\title{
Gastrectomy case volume and textbook outcome: an analysis of the Population Registry of Esophageal and Stomach Tumours of Ontario (PRESTO)
}

\author{
Jordan Levy ${ }^{1}$ (1) $\cdot$ Vaibhav Gupta $^{1}$ - Elmira Amirazodi ${ }^{2}$. Catherine Allen-Ayodabo ${ }^{2} \cdot$ Naheed Jivraj $^{3} \cdot$ Yunni Jeong $^{1}$.

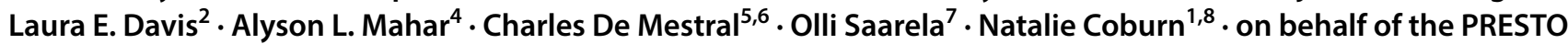 \\ Group
}

Received: 21 July 2019 / Accepted: 12 October 2019 / Published online: 4 November 2019

(c) The International Gastric Cancer Association and The Japanese Gastric Cancer Association 2019

\begin{abstract}
Objective To determine the association between gastric cancer surgery case-volume and Textbook Outcome, a new composite quality measurement.

Background Textbook Outcome included (a) negative resection margin, (b) greater than 15 lymph nodes sampled, (c) no severe complication, (d) no re-intervention, (e) no unplanned ICU admission, (f) length of stay of 21 days or less, (g) no 30-day readmission and (h) no 30-day mortality following surgery.

Methods All patients undergoing gastrectomy for non-metastatic gastric adenocarcinoma registered in the Population Registry of Esophageal and Stomach Tumours of Ontario between 2004 and 2015 were included. We used multivariable generalized estimating equation (GEE) logistic regression modelling to estimate the association between gastrectomy volume (surgeon and hospital annual volumes) and Textbook Outcome. Volumes were considered as continuous variables and quintiles. Results Textbook Outcome was achieved in 378 of 1660 patients $(22.8 \%)$. The quality metrics least often achieved were inadequate lymph node sampling and presence of severe complications, which occurred in $46.1 \%$ and $31.7 \%$ of patients, respectively. Accounting for covariates and clustering, neither surgeon volume nor hospital volume were significantly associated with Textbook Outcome. However, hospital volume was associated with adequate lymphadenectomy and fewer unplanned ICU admissions.

Conclusions Higher case volume can impact certain measures of quality of care but may not address all care structures necessary for ideal Textbook recovery. Future quality improvement strategies should consider using case-mix adjusted Textbook Outcome rates as a surgical quality metric.
\end{abstract}

Keywords Gastric cancer $\cdot$ Gastrectomy $\cdot$ Volume $\cdot$ Surgical quality $\cdot$ Textbook

The members of the PRESTO Groups are listed in the Acknowledgements section.

Electronic supplementary material The online version of this article (https://doi.org/10.1007/s10120-019-01015-w) contains supplementary material, which is available to authorized users.

Natalie Coburn

Natalie.Coburn@sunnybrook.ca

Extended author information available on the last page of the article

\section{Introduction}

Gastric cancer resections in North America often fall short of the standard of care. Population-based studies in the United States and Canada have reported positive resection margins in $6-11 \%$ of patients [1,2], inadequate lymph node sampling in $60-71 \%$ [3-6], and post-operative mortality rates of $5 \%[3,7]$.

Increased experience (volume) with gastric cancer surgery at the hospital- [8-11] and surgeon-level $[8,9,12]$ have both been associated with decreased operative mortality in multiple North American studies. However, death following surgery is an imperfect measure of surgical quality and represents just one of many factors important to patients, 
their families and their health care professionals. In 2017, a Dutch study combined 10 metrics to define high quality surgical care, henceforth entitled Textbook Outcome [13].

Achieving Textbook Outcome likely depends on expert decision-making, surgical technique and institutional processes of care. We hypothesized that centers and surgeons with greater experience in gastric cancer surgery are more likely to achieve a Textbook Outcome. The present study leverages the clinical and pathologic data in the Population Registry of Esophageal and Stomach Tumours of Ontario (PRESTO) to determine the association between gastric cancer surgery case-volume and Textbook Outcome rates.

\section{Methods}

\section{Study design and setting}

This is a population-based retrospective cohort study using health administrative data and a province-wide chart review of pathology reports. Adults who underwent gastrectomy for non-metastatic gastric adenocarcinoma in Ontario were included. Ontario is Canada's most populous province with over 14 million residents and a land mass greater than Texas and Arizona combined. In Ontario, all non-elective health services are funded through a universal and publicly administered health care system. Reporting is in accordance with the REporting of studies Conducted using Observational Routinely collected Data (RECORD) Statement [14] (Appendix 1).

\section{Data sources and management}

PRESTO is a population-based clinical-pathological database of all adult patients with esophagogastric cancer (International Classification of Disease for Oncology 3rd edition (ICD-O-3) topography codes "C15 and C16") of any histology, diagnosed and treated from 2002 onwards. It is derived from datasets linked using unique encoded identifiers and analyzed at ICES. ICES is an independent, nonprofit research institute whose legal status under Ontario's health information privacy law allows it to collect and analyze health care and demographic data, without consent, for health system evaluation and improvement. Tumor-specific information is available through the Esophagogastric Pathology Database which was populated by two physicians abstracting available operative specimen pathology reports for patients within PRESTO. Patients were identified through the Ontario Cancer Registry which captures $96 \%$ of incident cancer cases in Ontario [15, 16]. Patient demographics and vital status, cancer center treatment information, inpatient hospitalization records, physician billing data, diagnostic codes and interventional codes are available at ICES for every resident covered by Ontario's public health insurance plan.

\section{Study cohort}

This study included Ontario residents diagnosed with gastric adenocarcinoma undergoing curative-intent primary tumor resection between April 1, 2004 and March 31, 2015. Primary tumor site was defined according to gastric ICD-O-3 topography code "C16". ICD-O-3 morphological codes are included in Appendix 2. Curative-intent gastrectomy definition was based on the following rules: (a) patients must be electively admitted, (b) 0 days to 6 months following date of diagnosis, (c) first gastric resection and (d) no evidence of metastatic disease in the 6 months preceding surgery. Gastrectomies were identified using Canadian Classification of Intervention codes (Appendix 3). Patients with metastatic disease at time of surgery were identified using an algorithm validated in an Ontario gastric cancer cohort [17] and supplemented by the Esophagogastric Pathology Database stage variable. Patients were excluded if their date of death erroneously preceded their diagnosis date, if they were under the age of 18 years or if their resected tumor specimen pathology report was not available. Tumors at the gastroesophageal junction are often managed as esophageal cancers and esophageal surgery has been regionalized in Ontario since 2005. As such, we excluded patients with gastroesophageal junctional tumors undergoing esophagectomies as these would be overrepresented in high-volume hospitals.

\section{Exposure}

The main exposures in this study are hospital- and surgeonvolumes. Patients undergoing elective gastrectomies for adenocarcinomas 1 day to 6 months following diagnosis were identified through the hospital discharge abstract database intervention codes and were thus inherently linked to a hospital. Surgeons were identified through linkage to the provincial physician billing database using fee codes included in Appendix 4. To reduce any potential bias in penalizing low volume surgeons, we excluded physicians not identified as general surgeons, thoracic surgeons, cardiothoracic surgeons or vascular surgeons.

Volume was defined using the average yearly number of gastrectomy procedures performed in the 2 years prior to the date of the index procedure for each patient's surgeon and hospital [18]. Compared to averaging the number of procedures over the years of study, this definition accounts for year-to-year variation and excludes procedures performed subsequent to the index procedure, which should not affect the quality of care provided during the index procedure admission [19]. Volumes were also considered as categorical variables by ranking surgeons and hospitals in order of 
increasing annual volumes and selecting cut-points that most closely sorted patients into five evenly sized groups (quintiles $Q 1-Q 5$ ), similar to previously published studies $[9,11,18,20]$. This method avoids using arbitrary numbers from the literature which may not reflect the current state of care in this population. The unit of analysis in this study is the patient. In this regard, surgeon and hospital volumes are recorded for every patient, and specific surgeons and hospitals may have several different volumes (and volume quintiles) as these changed throughout the study period.

\section{Outcomes}

In this study, Textbook Outcome refers to a composite of eight quality metrics related to the oncologic resection, post-operative course and discharge of patients undergoing gastrectomy for cancer. These include (a) negative resection margins, (b) greater than 15 lymph nodes sampled, (c) no severe complications, (d) no re-interventions during index hospitalization, (e) no unplanned ICU admission, (f) length of stay (LOS) of 21 days or less, (g) no readmission to hospital in 30 days following discharge and (h) no mortality in the 30 days following surgery. Elaborated definitions are available in Appendix 5. Each of these metrics must be met to achieve Textbook Outcome. This concept was first proposed by the Dutch Upper Gastrointestinal Cancer Audit group [13] and we have modified it to conform to this analysis and the data available through ICES. Intent of surgery was included in the original Dutch study's definition but was felt to represent a significant selection bias in this study given that metastatic patients have less physiologic reserve, receive less extensive resections and are less likely to achieve Textbook Outcome. Intra-operative complications could not be unambiguously differentiated from post-operative complications within our datasets, and thus these were combined.

\section{Covariates}

Age at diagnosis (years), sex (male, female) and tumor location (ICD-O-3 topography codes C16.0-16.9) were available through the Ontario Cancer Registry. Resource utilization bands (RUB 0-5) were used in this study as a measure of baseline risk of death; these are available through the Johns Hopkins Adjusted Clinical Groups ${ }^{\circledR}$ System (Version 10.0.1) which is a validated predictor of 1 -year mortality and used for comorbidity adjustment [21]. Material deprivation (quintile 1-5), available through the census-based, geographically derived Ontario Marginalization Index (ON-Marg) [22] was used as a measure of socioeconomic status and is based on income, quality of housing, educational attainment, and family structure characteristics. Year of gastrectomy (2004-2015) was based on the date of the gastrectomy intervention code in the
Canadian Classification of Health Intervention codes. Histology category (intestinal vs. non-intestinal) was based on the ICD-O-3 morphological codes available through the Ontario Cancer Registry and supplemented by the Esophagogastric Pathology Database when the pathologist reported on findings of linitis plastica, diffuse and/or signet ring cell histology. Pathologic $\mathrm{T}$ and $\mathrm{N}$ stages were available through the esophagogastric pathology database.

\section{Statistical analysis}

Patient baseline demographics and tumor characteristics of the highest surgeon quintile $(Q 5)$ and the lowest surgeon volume quintile $(Q 1)$ were compared using standardized differences (SD). This was repeated using hospital volume quintiles.

A multivariable generalized estimating equation (GEE) logistic model was specified to characterize the association between surgeon volume (continuous distribution) and Textbook Outcome as the odds ratio of achieving Textbook Outcome with increasing surgeon volume. The model accounted for clustering by surgeon and adjusted for patient-level confounders. Covariates were selected a priori as potential confounders based on clinical grounds. These covariates were assessed for statistical collinearity using a variation inflation factor cut-off of four. This was repeated with hospital volume (continuous distribution). To estimate the association between surgeon and hospital volumes and Textbook Outcome, both volume variables were subsequently included in the model. Naïve model (non-GEE) calibration and discrimination were assessed using the Hosmer and Lemeshow Goodness-of-Fit test (satisfactory fit if $p>0.05$ ) and the "c" statistic, respectively [23].

For ease of interpretation, this was repeated using surgeon volume quintiles (Surgeon $Q 5$ vs. $Q 1$ ) and hospital volume quintiles (Hospital $Q 5$ vs. $Q 1$ ). Correlation between surgeon and hospital volumes as continuous variables was determined using the Spearman Correlation Coefficient (significant if $p<0.05$ ). Correlation between surgeon and hospital volumes as categorical quintiles was determined using the polychoric correlation coefficient (significant if $p<0.05$ ). To elucidate the associations between surgeon volume quintiles, hospital volume quintiles and the Textbook Outcome metrics themselves, identical models were fitted with both volume quintile variables and covariates for each TO metric independently. Statistical analyses were performed using SAS v9.4 (SAS Institute Inc., Cary, NC, USA). All tests were two-sided with $p$ values of $<0.05$ considered statistically significant and standardized differences greater than $10 \%$ considered clinically meaningful. 


\section{Missing data}

A substantial amount of the data used in this study are available through validated health administrative databases [24, 25] where missing data is quite rare (majority $<1 \%$ ) [26]. However, many gastrectomy patients were missing pathology reports necessary for assessing margin status, lymph node sampling and stage. As such, a mixed effects multivariable logistic regression accounting for clustering by hospital was used to determine whether gastrectomy volume and TO were associated with pathology report missingness. All available covariates included in the primary analysis model were included in the missing pathology data model.

\section{Ethical approval}

The use of data in this project was authorized under Section 45 of Ontario's Personal Health Information Protection Act. This study was approved by the research ethics board of Sunnybrook Health Sciences Centre and underwent privacy review at ICES.

\section{Results}

\section{Study cohort}

There were 2911 patients diagnosed with gastric adenocarcinoma who underwent an eligible resection during the study period. Of these, 2151 (74\%) had a pathology report available for review and these patients were included in the study. Compared to the those excluded based on missing pathology report, those with pathology reports were slightly older (mean age 67.9 vs. 65.9, standardized difference (SD)
$16 \%$ ) and were more contemporary (median year of gastrectomy 2010 vs. 2008, SD 35\%), but were otherwise similar in sex, socioeconomic status, baseline health status, and tumor location (with the exception of tumor location "Gastric NOS" which was less common in the included patients, potentially due to improved tumor location capture in more recent years). Pathology report missingness was significantly associated with hospital volume, such that patients at lowest volume hospitals were twice as likely to be missing a pathology report than those at the highest volume hospitals (Hospital $Q 1$ vs. $Q 5-$ a OR 2.10 [1.42-3.10], $p<0.001$ ). However, missingness was not associated with surgeon volume, severe complications, reinterventions, unplanned ICU admissions, prolonged LOS, 30-day readmissions or 30-day mortality. As such, missingness of pathology reports was not deemed a confounder in this analysis. Patients with metastatic disease $(n=259)$ and those with gastroesophageal junctional tumors $(n=150)$ were excluded. Of the remaining 1742 eligible patients, 1707 (98\%) were successfully linked to a surgeon using the physician billing database. Finally, following exclusion of patients with missing baseline demographic or tumor characteristic data (3\%), a study cohort of 1660 patients was available for analysis (Appendix 6).

\section{Textbook outcome}

Textbook Outcome was achieved in 378 of 1660 patients (22.8\%). The quality metrics least often achieved were inadequate lymph node sampling and presence of severe complications, which occurred in $46.1 \%$ and $31.7 \%$ of patients, respectively. The distribution of each quality metric and their effect on the TO rate are presented in Fig. 1. The proportion achieving Textbook Outcome varied significantly by year of surgery and displayed a significant and positive trend
Fig. 1 Proportion and cumulative incidence of patients achieving each quality metric and textbook outcome

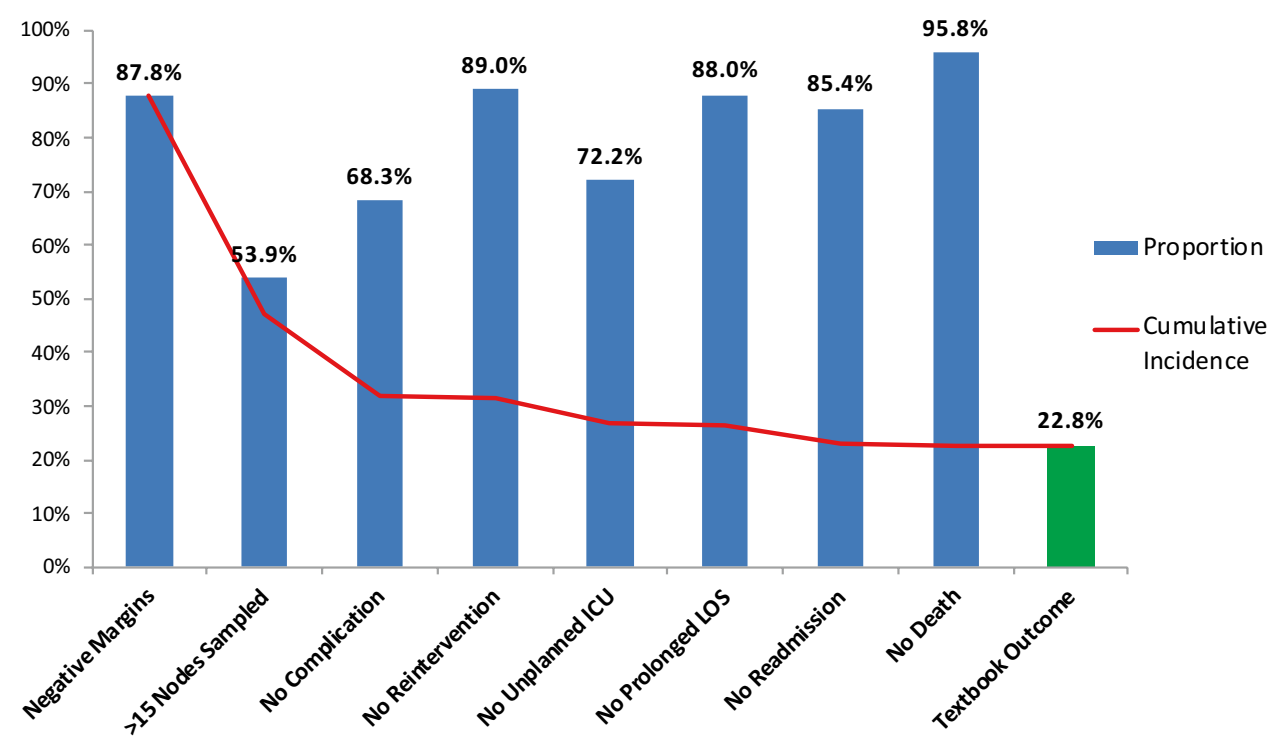


(Cochran-Armitage Trend test, $p<0.001$ ); it was $20.3 \%$ in 2004 and 29.3\% in 2015 (Appendix 7). On adjusted GEE logistic regression, each subsequent year from 2004 to 2015 was independently associated with an $8 \%$ increase in the odds of achieving Textbook Outcome (aOR 1.08 [1.03-1.13], $p=0.001$ ); patients undergoing gastrectomy in 2015 were $88 \%$ more likely to achieve it than patients in 2004.

\section{Gastrectomy volumes}

The number of patients, surgeons, hospitals, volume cut points and median volumes for each volume-patient group are presented in Table 1 . There were 355 surgeons operating at 69 hospitals. The highest and lowest volume quintile surgeons performed 3.5-9.5 and 0 gastrectomies per year, respectively. The highest and lowest volume quintile hospitals performed $12-22$ and $0-2$ gastrectomies per year, respectively. Surgeons performed a median of one case per year, and hospitals performed a median of 6.5 cases per year. The highest volume surgeons, or those in the top quintile, performed a median of 4.5 cases annually. The highest volume hospitals performed a median of 14.5 cases annually. Patients were more likely to undergo surgery by the lowest volume surgeons if they went to the lowest volume hospitals than if they went to the highest volume hospitals (38.5\% vs.
12.4\%). Baseline characteristics and statistical comparisons using standardized differences between patients treated by the highest compared to the lowest volume surgeons (surgeon $Q 5$ vs. $Q 1)$ and between those treated at the highest compared to the lowest volumes hospitals (hospital $Q 5$ vs. $Q 1)$ are presented in Tables 2 and 3. Patients in the highest surgeon and hospital quintile groups were treated more recently and were more likely to have proximal tumors (gastric cardia or fundus). Patients treated at the highest volume hospitals were more comorbid (elevated RUB) and of lower socioeconomic status (elevated material deprivation quintile).

\section{Textbook outcome rates across gastrectomy volumes}

Achieving Textbook Outcome was rare across all surgeons (median rate $0 \%$, interquartile range (IQR) $0-33 \%$ ) and all hospitals (median rate 17\%, IQR 0-28\%). Textbook Outcome was achieved in a similar proportion of the patients treated by the highest volume surgeons compared to the lowest volume surgeons (Surgeon Q5 24.0\% vs. Q1 20.8\%, SD $8 \%$ ) (Table 4). Adequate lymph node sampling and absence of severe complications metrics were superior in the highest volume surgeon group, but this was counteracted by the higher reintervention rate in this group.

Table 1 Surgeon and hospital volume quintiles

\begin{tabular}{|c|c|c|c|c|c|}
\hline Surgeon volume quintiles & $Q 1$ & $Q 2$ & $Q 3$ & $Q^{4}$ & $Q 5$ \\
\hline Number of patients & 356 & 296 & 368 & 319 & 321 \\
\hline Number of surgeons & 277 & 194 & 154 & 85 & 36 \\
\hline Annual volume cut points & 0 & 0.5 & $1.0-1.5$ & $2.0-3.0$ & $3.5-9.5$ \\
\hline Annual volume, median (IQR) & $0(0)$ & $0.5(0.5)$ & $1(1.0-1.5)$ & $2.5(2.0-3.0)$ & $4.5(4.0-6.0)$ \\
\hline Number of hospitals & 67 & 52 & 47 & 39 & 24 \\
\hline \multicolumn{6}{|l|}{ Hospital volume quintiles, $n(\%)$} \\
\hline$Q 1$ & $137(38.5 \%)$ & $68(23.0 \%)$ & $50(13.6 \%)$ & $78^{\mathrm{a}}(24.4 \%)$ & $28^{\mathrm{a}}(8.7 \%)$ \\
\hline$Q^{2}$ & $82(23.0 \%)$ & $85(28.7 \%)$ & $107(29.1 \%)$ & & \\
\hline$Q 3$ & $41(11.5 \%)$ & $48(16.2 \%)$ & $81(22.0 \%)$ & $67(21.0 \%)$ & $71(22.1 \%)$ \\
\hline$Q 4$ & $52(14.6 \%)$ & $49(16.6 \%)$ & $81(22.0 \%)$ & $100(31.4 \%)$ & $111(34.6 \%)$ \\
\hline$Q 5$ & $44(12.4 \%)$ & $46(15.5 \%)$ & $49(13.3 \%)$ & $74(23.2 \%)$ & $111(34.6 \%)$ \\
\hline Hospital volume quintiles & $Q 1$ & $Q 2$ & $Q 3$ & $Q 4$ & $Q 5$ \\
\hline Number of patients & 259 & 376 & 308 & 393 & 324 \\
\hline Number of Hospitals & 64 & 42 & 31 & 22 & 12 \\
\hline Annual volume cut points & $0-2.0$ & $2.5-5.0$ & $5.5-7.5$ & $8.0-11.5$ & $12.0-22.0$ \\
\hline Annual volume, median (IQR) & $1.0(0.5-1.5)$ & $4.0(3.0-4.5)$ & $6.5(6.0-7.0)$ & $9.5(8.5-10.5)$ & $14.5(12.5-16.5)$ \\
\hline Number of surgeons & 147 & 164 & 122 & 110 & 79 \\
\hline
\end{tabular}

Caption: Patients included in the study cohort were divided into five approximately equally-sized patient groups based on their surgeon's annual volume (surgeon volume quintiles $Q 1-Q 5$ ) and their hospital's annual volume (hospital volume quintiles $Q 1-Q 5$ )

$I Q R$ interquartile range

${ }^{\text {a}}$ Hospital Quartiles 1 and 2 are combined to avoid publication of cells with less than 6 patients, as per ICES' Re-identification risk assessment procedure policy 
Table 2 Comparison of baseline characteristics for patients treated by the highest vs. lowest volume surgeons (surgeon volume quintile 5 vs. 1)

\begin{tabular}{|c|c|c|c|}
\hline Characteristic & Surgeon volume $Q 1$ & Surgeon volume $Q 5$ & $\mathrm{SD}(\%)$ \\
\hline \multicolumn{4}{|l|}{ Age groups, $n(\%)$} \\
\hline$<50$ & $32(9.0)$ & $29(9.0 \%)$ & 0 \\
\hline $50-59$ & $44(12.4 \%)$ & $54(16.8 \%)$ & 13 \\
\hline $60-69$ & $94(26.4 \%)$ & $67(20.9 \%)$ & 13 \\
\hline $70-79$ & $121(34.0 \%)$ & $114(35.5 \%)$ & 3 \\
\hline$>80$ & $65(18.3 \%)$ & $57(17.8 \%)$ & 1 \\
\hline \multicolumn{4}{|l|}{ Sex, $n(\%)$} \\
\hline Female & $123(34.6 \%)$ & $107(33.3 \%)$ & 3 \\
\hline Male & $233(65.4 \%)$ & $214(66.7 \%)$ & 3 \\
\hline \multicolumn{4}{|l|}{ Resource utilization band, $n(\%)$} \\
\hline $0-2$ & $17(4.8 \%)$ & $11(3.4 \%)$ & 7 \\
\hline 3 & $166(46.6 \%)$ & $156(48.6 \%)$ & 4 \\
\hline 4 & $109(30.6 \%)$ & $88(27.4 \%)$ & 7 \\
\hline 5 & $64(18.0 \%)$ & $66(20.6 \%)$ & 7 \\
\hline \multicolumn{4}{|l|}{ Material deprivation quintile, $n(\%)$} \\
\hline 1 & $54(15.2 \%)$ & $49(15.3 \%)$ & 0 \\
\hline 2 & $65(18.3 \%)$ & $54(16.8 \%)$ & 4 \\
\hline 3 & $70(19.7 \%)$ & $61(19.0 \%)$ & 2 \\
\hline 4 & $81(22.8 \%)$ & $74(23.1 \%)$ & 1 \\
\hline 5 & $86(24.2 \%)$ & $83(25.9 \%)$ & 4 \\
\hline Year of gastrectomy, median (IQR) & 2009 (2007-2012) & $2010(2008-2013)$ & 34 \\
\hline \multicolumn{4}{|l|}{ Tumor location, $n(\%)$} \\
\hline Cardia, NOS & $24(6.7 \%)$ & $41(12.8 \%)$ & 20 \\
\hline Fundus & $8(2.2 \%)$ & $20(6.2 \%)$ & 20 \\
\hline Body & $50(14.0 \%)$ & $47(14.6 \%)$ & 2 \\
\hline Antrum & $155(43.5 \%)$ & $106(33.0 \%)$ & 22 \\
\hline Pylorus & $18(5.1 \%)$ & $9(2.8 \%)$ & 12 \\
\hline Lesser curvature, NOS & $53(14.9 \%)$ & $45(14.0 \%)$ & 2 \\
\hline Greater curvature, NOS & $14(3.9 \%)$ & $10(3.1 \%)$ & 4 \\
\hline Overlapping lesion & $7(2.0 \%)$ & $8(2.5 \%)$ & 4 \\
\hline NOS & $27(7.6 \%)$ & $35(10.9 \%)$ & 11 \\
\hline \multicolumn{4}{|l|}{ Histology category, $n(\%)$} \\
\hline Intestinal & $227(63.8 \%)$ & $203(63.2 \%)$ & 1 \\
\hline Non-intestinal & $129(36.2 \%)$ & $118(36.8 \%)$ & 1 \\
\hline \multicolumn{4}{|l|}{ T-stage, $n(\%)$} \\
\hline T0-1 & $86(24.2 \%)$ & $90(28.0 \%)$ & 9 \\
\hline $\mathrm{T} 2$ & $66(18.5 \%)$ & $47(14.6 \%)$ & 10 \\
\hline $\mathrm{T} 3$ & $101(28.4 \%)$ & $94(29.3 \%)$ & 2 \\
\hline $\mathrm{T} 4 \mathrm{a}$ & $92(25.8 \%)$ & $78(24.3 \%)$ & 4 \\
\hline $\mathrm{T} 4 \mathrm{~b}$ & $11(3.1 \%)$ & $12(3.7 \%)$ & 4 \\
\hline \multicolumn{4}{|l|}{ Node involvement, $n(\%)$} \\
\hline No & $143(40.2 \%)$ & $129(40.2 \%)$ & 0 \\
\hline $\mathrm{N}+$ & $213(59.8 \%)$ & $192(59.8 \%)$ & 0 \\
\hline
\end{tabular}

Bold values indicate standardized Difference of $10 \%$ or greater considered clinically meaningful $S D$ standardized differences, $I Q R$ interquartile range
Textbook Outcome was achieved in a higher proportion of the patients treated at the highest volume hospitals compared to the lowest volume hospitals (Hospital Q5 $23.5 \%$ vs. $Q 116.2 \%$, SD $18 \%$ ) (Table 5). This difference was driven by the adequate lymph node sampling rate, the lower rate of unplanned ICU admissions and lower rates of 30-day mortality. The reintervention rate remained higher in this highest volume hospital group. 
Table 3 Comparison of baseline characteristics for patients treated at the highest vs. lowest volume hospitals (hospital volume quintile 5 vs. 1)

\begin{tabular}{|c|c|c|c|}
\hline Characteristic & Hospital volume $Q 1$ & Hospital volume $Q 5$ & $\mathrm{SD}(\%)$ \\
\hline \multicolumn{4}{|l|}{ Age groups, $n(\%)$} \\
\hline$<50$ & $16(6.2 \%)$ & $31(9.6 \%)$ & 13 \\
\hline $50-59$ & $35(13.5 \%)$ & $53(16.4)$ & 8 \\
\hline $60-69$ & $70(27.0 \%)$ & $78(24.1 \%)$ & 7 \\
\hline $70-79$ & $86(33.2 \%)$ & $104(32.1 \%)$ & 2 \\
\hline$>80$ & $52(20.1 \%)$ & $58(17.9 \%)$ & 6 \\
\hline \multicolumn{4}{|l|}{ Sex, $n(\%)$} \\
\hline Female & $99(38.2 \%)$ & $101(31.2 \%)$ & 15 \\
\hline Male & $160(61.8 \%)$ & $223(68.8 \%)$ & 15 \\
\hline \multicolumn{4}{|l|}{ Resource utilization band, $n(\%)$} \\
\hline $0-2$ & $8(3.1 \%)$ & $17(5.2 \%)$ & 11 \\
\hline 3 & $125(48.3 \%)$ & $152(46.9 \%)$ & 3 \\
\hline 4 & $87(33.6 \%)$ & $84(25.9 \%)$ & 17 \\
\hline 5 & $39(15.1 \%)$ & $71(21.9 \%)$ & 18 \\
\hline \multicolumn{4}{|l|}{ Material deprivation quintile, $n(\%)$} \\
\hline 1 & $49(18.9 \%)$ & $46(14.2 \%)$ & 13 \\
\hline 2 & $54(20.8 \%)$ & $50(15.4 \%)$ & 14 \\
\hline 3 & $53(20.5 \%)$ & $64(19.8 \%)$ & 2 \\
\hline 4 & $50(19.3 \%)$ & $76(23.5 \%)$ & 10 \\
\hline 5 & $53(20.5 \%)$ & $88(27.2 \%)$ & 16 \\
\hline Year of gastrectomy, median (IQR) & 2008 (2005-2012) & $2010(2008-2012)$ & 50 \\
\hline \multicolumn{4}{|l|}{ Tumor location, $n(\%)$} \\
\hline Cardia/fundus* & $19(7.3 \%)$ & $65(20.1 \%)$ & 25 \\
\hline Body & $32(12.4 \%)$ & $40(12.3 \%)$ & 0 \\
\hline Antrum & $104(40.2 \%)$ & $115(35.5 \%)$ & 10 \\
\hline Pylorus & $12(4.6 \%)$ & $9(2.8 \%)$ & 10 \\
\hline Lesser curvature, NOS & $40(15.4 \%)$ & $52(16.0 \%)$ & 2 \\
\hline Greater curvature, NOS & $15(5.8 \%)$ & $16(4.9 \%)$ & 4 \\
\hline Overlapping lesion & $10(3.9 \%)$ & $9(2.8 \%)$ & 6 \\
\hline NOS & $27(10.4 \%)$ & $18(5.6 \%)$ & 18 \\
\hline \multicolumn{4}{|l|}{ Histology category, $n(\%)$} \\
\hline Intestinal & $161(62.2 \%)$ & $199(61.4 \%)$ & 2 \\
\hline Non-intestinal & $98(37.8 \%)$ & $125(38.6 \%)$ & 2 \\
\hline \multicolumn{4}{|l|}{ T-stage, $n(\%)$} \\
\hline T0-1 & $65(25.1 \%)$ & $71(21.9 \%)$ & 8 \\
\hline $\mathrm{T} 2$ & $44(17.0 \%)$ & $56(17.3 \%)$ & 1 \\
\hline $\mathrm{T} 3$ & $71(27.4 \%)$ & $107(33.0 \%)$ & 12 \\
\hline $\mathrm{T} 4 \mathrm{a}$ & $62(23.9 \%)$ & $79(24.4 \%)$ & 1 \\
\hline $\mathrm{T} 4 \mathrm{~b}$ & $17(6.6 \%)$ & $11(3.4 \%)$ & 15 \\
\hline \multicolumn{4}{|l|}{ Node involvement, $n(\%)$} \\
\hline No & $143(55.2 \%)$ & $188(58.0 \%)$ & 6 \\
\hline $\mathrm{N}+$ & $116(44.8 \%)$ & $136(42.0 \%)$ & 6 \\
\hline
\end{tabular}

Bold values indicate standardized difference of $10 \%$ or greater considered clinically meaningful $S D$ standardized differences, $I Q R$ interquartile range

*Cardia and Fundus combined to avoid publication of small cells $(<6)$ according to privacy policy
On unadjusted logistic regression, surgeon volume as a continuous variable $(p=0.20)$ and hospital volume as a continuous variable $(p=0.09)$ were not associated with Textbook Outcome. When surgeon $(p=0.46)$ and hospital $(p=0.19)$ volumes were both included together as continuous variables in the model, neither was associated with Textbook Outcome. Given the marked heterogeneity in the surgeon and hospital volume quintile groups and potential for 
Table 4 Comparison of textbook outcome and metrics for patients treated by the highest vs. lowest volume surgeons (surgeon volume quintile 5 vs. 1)
Table 5 Comparison of textbook outcome and metrics for patients treated at the highest vs. lowest volume hospitals (hospital volume quintile 5 vs. 1)

\begin{tabular}{lllc}
\hline Characteristic & Surgeon volume $Q 1$ & Surgeon volume $Q 5$ & SD (\%) \\
\hline Textbook outcome & $20.8 \%$ & $24.0 \%$ & 8 \\
Negative margins & $89.6 \%$ & $88.5 \%$ & 4 \\
Greater than 15 LN Sampled & $45.5 \%$ & $62.0 \%$ & $\mathbf{3 4}$ \\
Number of nodes sampled, median (IQR) & $14(8-20)$ & $19(13-29)$ & $\mathbf{5 3}$ \\
No severe complication & $69.1 \%$ & $74.1 \%$ & $\mathbf{1 1}$ \\
No reintervention & $93.3 \%$ & $88.5 \%$ & $\mathbf{1 7}$ \\
No unplanned ICU admission & $75.0 \%$ & $71.0 \%$ & 9 \\
Length of stay 21 days or Less & $88.5 \%$ & $89.7 \%$ & 4 \\
Length of stay (days), median (IQR) & $9(7-13)$ & $9(7-13)$ & 9 \\
No 30-day readmission & $83.4 \%$ & $82.6 \%$ & 2 \\
No 30-day mortality & $96.6 \%$ & $95.3 \%$ & 7 \\
\hline
\end{tabular}

Bold values indicate standardized difference of $10 \%$ or greater considered clinically meaningful $S D$ standardized differences, $I Q R$ interquartile range

\begin{tabular}{lllr}
\hline Characteristic & Hospital volume $Q 1$ & Hospital volume $Q 5$ & SD (\%) \\
\hline Textbook outcome & $16.2 \%$ & $23.5 \%$ & $\mathbf{1 8}$ \\
Negative margins & $84.9 \%$ & $84.9 \%$ & 0 \\
Greater than 15 LN sampled & $38.2 \%$ & $62.0 \%$ & $\mathbf{4 9}$ \\
Number of nodes sampled, median (IQR) & $13(8-20)$ & $18(13-25)$ & $\mathbf{5 9}$ \\
No severe complication & $67.2 \%$ & $69.4 \%$ & 5 \\
No reintervention & $91.5 \%$ & $86.7 \%$ & $\mathbf{1 5}$ \\
No unplanned ICU admission & $66.8 \%$ & $80.2 \%$ & $\mathbf{3 1}$ \\
Length of stay 21 days or less & $88.0 \%$ & $88.0 \%$ & 0 \\
Length of stay (Days), median (IQR) & $9(7-13)$ & $9(7-12)$ & $\mathbf{1 1}$ \\
No 30-day readmission & $83.8 \%$ & $80.2 \%$ & 9 \\
No 30-day mortality & $93.4 \%$ & $96.9 \%$ & $\mathbf{1 6}$
\end{tabular}

Bold values indicate standardized difference of $10 \%$ or greater considered clinically meaningful $S D$ standardized differences, $I Q R$ interquartile range clustering by surgeon, a multivariable generalized estimating equation (GEE) logistic model was fitted to patient age, sex, RUB, deprivation quintile, year of gastrectomy, tumor location, histology category and $\mathrm{T}$ and $\mathrm{N}$ stage. Continuous surgeon volumes $(p=0.41)$ and continuous hospital volumes $(p=0.24)$ were not significantly associated with Textbook Outcome. Continuous volumes were moderately correlated (Spearman Correlation Coefficient 41\%, $p<0.001$ ) and not collinear $(\mathrm{VIF}=1.19)$. When both were included together, neither surgeon $(p=0.67)$ nor hospital $(p=0.33)$ volume as continuous variables, was associated with Textbook Outcome.

Quintile volumes were moderately correlated (Polychoric Correlation Coefficient 45\%, $p<0.001$ ) and not collinear (VIF $=1.26)$. Compared to the lowest volume surgeons, patients treated by the highest volume surgeons had similar outcomes (Surgeon $Q 5$ vs. $Q 1-a O R ~ 1.18$ [0.78-1.78], $p=0.42$ ). Compared to the lowest volume hospitals, patients treated at the highest volume hospitals were $45 \%$ more likely to achieve Textbook Outcome, however this difference was not statistically significant (Hospital $Q 5$ vs. $Q 1-a O R 1.45$ [0.93-2.29], $p=0.10$ ). When both quintile volumes were included in the model, neither surgeon volume quintile (Surgeon $Q 5$ vs. $Q 1$-aOR 1.05 [0.68-1.62], $p=0.82$ ) nor hospital volume quintile (Hospital $Q 5$ vs. $Q 1-\mathrm{aOR} 1.37$ [0.84-2.22], $p=0.21$ ) was associated with Textbook Outcome (Appendix 8).

\section{Textbook outcome rates across combined gastrectomy volumes}

Using GEE logistic regression adjusted for covariates and clustering by surgeon, the association of both surgeon and hospital volume quintiles with each Textbook Outcome metric was estimated (Table 6). Compared to the lowest volume hospitals, patients treated at the highest volume hospitals were $92 \%$ more likely to have adequate lymphadenectomy (Hospital $Q 5$ vs. $Q 1-a O R 1.92$ [1.24-2.97], $p=0.003$ ) and 
Table 6 Association between surgeon and hospital volume quintiles and textbook outcome metrics (adjusted odds ratios and 95\% CI)

\begin{tabular}{|c|c|c|c|c|c|c|}
\hline Characteristic & Surgeon volume $Q 1$ & Surgeon volume $Q 5$ & $p$ value & Hospital volume $Q 1$ & Hospital volume $Q 5$ & $p$ value \\
\hline Textbook outcomes & Reference & $1.05(0.68-1.62)$ & 0.82 & Reference & $1.37(0.84-2.22)$ & 0.21 \\
\hline Negative margins & Reference & $0.96(0.48-1.93)$ & 0.92 & Reference & $0.98(0.55-1.73)$ & 0.94 \\
\hline Greater than $15 \mathrm{LN}$ sampled & Reference & $1.33(0.84-2.10)$ & 0.22 & Reference & $1.92(1.24-2.97)$ & 0.003 \\
\hline No severe complication & Reference & $1.38(0.82-2.32)$ & 0.22 & Reference & $1.11(0.70-1.76)$ & 0.65 \\
\hline No reintervention & Reference & $0.72(0.37-1.40)$ & 0.33 & Reference & $0.71(0.36-1.42)$ & 0.33 \\
\hline No unplanned ICU admission & Reference & $0.66(0.39-1.12)$ & 0.13 & Reference & $3.23(1.97-5.31)$ & $<0.001$ \\
\hline Length of stay 21 days or less & Reference & $1.18(0.69-2.03)$ & 0.54 & Reference & $0.91(0.50-1.67)$ & 0.76 \\
\hline No 30-day readmission & Reference & $0.94(0.58-1.52)$ & 0.79 & Reference & $0.85(0.52-1.37)$ & 0.50 \\
\hline No 30-day mortality & Reference & $0.96(0.64-1.45)$ & 0.86 & Reference & $0.73(0.46-1.17)$ & 0.19 \\
\hline
\end{tabular}

95\% CI 95\% confidence intervals

Multivariable generalized estimating equation logistic model adjusted for surgeon and hospital volume quintiles, covariates and clustering by surgeon

$323 \%$ more likely to avoid an unplanned ICU admission (Hospital $Q 5$ vs. $Q 1-$ aOR 3.23 [1.97-5.31], $p<0.001$ ). Surgeon and hospital volume quintiles were not significantly associated with any of the other metrics.

\section{Discussion}

This study provides evidence that measuring Textbook Outcome is feasible and informative. Cancer surgery quality is multidimensional and generating population data on Textbook Outcome provides a comprehensive overview of the current state of surgical management of gastric cancer. Gastrectomies are an invasive procedure with important rates of post-operative complications and mortality. Patients benefit most when the procedure is safe and effective at reducing the risk of local recurrence.

Textbook Outcome was achieved in $22.8 \%$ of the study cohort, lower than the proportion of $32.1 \%$ reported in the original Dutch study [13]. This was not unexpected, given the regionalization of gastric surgery in the Netherlands since 2012, and no formal gastric cancer regionalization strategy in Ontario. The quality metric that had the most negative impact in both studies was the adequacy of lymph node harvest, which occurred in only $53.9 \%$ of Canadian and $57.1 \%$ of Dutch patients. Where the results of this study differed most from the Dutch study was the proportion of patients with severe complications $(31.7 \%$ vs. $11.7 \%)$ and unplanned admissions to the ICU $(27.8 \%$ vs. 9\%). Patients undergoing gastrectomy at the highest volume hospitals were greater than three times more likely to avoid an unplanned ICU admission than those treated at the lowest volume hospitals. Despite decreased ICU admissions, complication rates were the same across these hospital groups. These results substantiate a previous volume study, which found that hospitals in the highest volume quintile ( $>11$ annual gastrectomy cases) had superior rescue rates, but similar complication rates [27].

While Textbook Outcome was not associated with either surgeon or hospital volumes, we did find that in addition to decreasing unplanned ICU admissions, the highest volume hospitals had improved adequate lymphadenectomy rates. When hospital volume was accounted for in the model, surgeon volume was no longer associated with adequate lymphadenectomy rates. Highest volume hospitals resected a median of 18 nodes, and at least 16 nodes in $62 \%$. Lowest volume hospitals resected a median of 13 nodes, and at least 16 nodes in $38 \%$. This may speak to the multidisciplinary nature of lymph node harvest that is not uniquely dependent on surgeon ability, but a collaboration between surgeons and pathologists. This is a very important finding, given the evidence from the colorectal cancer literature that specimen processing [28] and collaborative educational-programs for surgeons and pathologists [29] can significantly increase lymph node harvest in oncologic resections. These types of programs may represent yet another institutional policy that may greatly benefit not only patients with gastric cancer, but nearly all solid tumor malignancies with staging dependent on lymph node status.

The evidence supporting increased surgeon and hospital volumes stems from multiple North American studies that have shown decreased gastrectomy associated mortality with increased center experience (hospital volume) [8-11,30] and surgeon experience (surgeon volume) $[8,9]$. However, these correlations have not been consistently shown at the hospital [31, 32] or surgeon-level [12]. In addition to the association with post-operative morality, one US state-wide analysis found that greater hospital-volume was also associated with decreased length of stay and cost in gastric cancer surgery [32], while a National Cancer Database analysis found that higher-volume hospitals examined more lymph 
nodes [33]. The latter is corroborated by the results of the present study.

Textbook Outcome rates increased significantly during the study period; patients undergoing gastrectomy in 2015 were $88 \%$ more likely to achieve it than patients in 2004 . Despite identifying a natural regionalization of gastrectomies over the study period - the median years of gastrectomies performed by the highest and lowest volume surgeons were 2010 and 2009, and the median years of gastrectomies performed at the highest and lowest volume hospitals were 2010 and 2008-this time-dependent improvement in TO rates was independent of gastrectomy volume. In Ontario, there has been government-backed regionalization policies for thoracic surgery since 2005 and hepatopancreatobiliary surgery since $2006[34,35]$. The association between regionalization of related complex cancer surgeries and the increase in Textbook Outcome rates in gastric cancer over time is speculative, but there is evidence of non-specific surgical volumes and improved outcomes in lung, esophageal, colorectal resections and repair of aortic aneurysms in Ontario [36].

The gastrectomy cases included in the volume calculation are restricted to gastrectomies performed in the elective setting, subsequent to a diagnosis of gastric adenocarcinoma and within 6 months of this diagnosis. In the low incidence region of North America, a high-volume surgeon has been previously defined as one performing greater than 3 resections annually $[9,12]$, whereas a high volume center has been defined according to a cut-off between 15 and 35 annual cases [8-11, 30, 31, 37]. A recent international and multidisciplinary expert panel [38] has provided consensus guidelines on the processes of care for gastric cancer, and has deemed it necessary for practicing surgeons to be trained in gastric cancer surgery and appropriate for them to perform greater than 6 cases annually at centers with case volumes greater than 15 annually. As such, the volumes of the highest volume surgeons and hospitals reported in this study are in line with previous studies performed in North America and recent consensus guidelines. Furthermore, D2 lymphadenectomies are complex procedures, with a learning curve as high as 15-100 cases before a plateau is reached and varies according to what parameters are measured [39, 40]. Given the current volume patterns in Ontario, it would take the highest volume surgeons many years to achieve competency, whereas surgeons performing less than two annual cases (Surgeon Volume Quintile 1-3) may never attain this goal.

Centralization of a rare procedure is important for specialists to achieve sufficient experience to develop and maintain their expertise, for advancing medical knowledge through research and allows for mobilizing human resources and physical structures necessary for achieving high quality surgery [41], for example through standardized clinical pathways and availability of ICU and interventional radiology physicians. It is our opinion that centralization of gastric cancer surgery should happen, but that simply concentrating the number of cases per hospital or surgeon is not enough. What is of equal importance is the need to improve care when regionalizing. The volume-outcome relationship is akin to "practice makes perfect", when in fact "perfect practice makes perfect" may be more appropriate [41]. Future policies should be more aligned to meeting quality metrics than an absolute volume. Textbook Outcome and other outcome metrics could be used to identify surgeons with excellent gastric cancer surgery outcomes to serve as mentors, to participate in the centralization of gastric cancer care, and as a means to identify areas requiring improvements for all surgeons and the processes of care available to them. Surgicopathologic quality (margins and lymph nodes) in this study was poor and represents a combined responsibility between surgeons and pathologists [28, 29]. Intraoperative frozen section margin is highly accurate [42], but requires the presence of a timely and efficient pathology review. Achieving adequate lymph node numbers depend on adequate resection in addition to specimen processing and evaluation. D2 lymphadenectomy $[38,43,44]$ is recommended by several guidelines, but true uptake in our study and several other North American population studies remains low based on lymph node count [3-6]. In low volume countries, specialized fellowship training, D2 gastrectomy courses [45], or international observerships could be utilized to improve outcomes, and the use of Textbook Outcome and its metrics as a means of objective and actionable feedback can be leveraged to continue improving outcomes after centralization. Regrettably, providing metrics to surgeons and hospitals will only be meaningful to those with an adequate volume, thus reinforcing the need to centralize rare procedures [46].

This study is a large, population-based analysis designed to capture all patients who underwent a potentially curative gastrectomy for gastric adenocarcinoma in Ontario. The strengths of this study are based on its stringent methods, expansive data linkage and granular pathology data. Compared to institutional-series and clinical trials, the findings in this study are more widely generalizable and represent Ontario's real-world experience with gastric cancer surgery. Extensive collaboration with our provincial cancer registry and a significant investment in pathology report extraction has allowed for a unique opportunity to leverage pathology specimen data to measure surgical quality outcomes and account for cancer-specific confounders. Unfortunately, $26 \%$ of eligible patients were lacking a pathology report, despite significant attempts to acquire them. We used statistical modelling to determine that missingness of a pathology report was not a confounder in the gastrectomy volume and Textbook Outcome relationship. A second limitation to this study was the inability to conclusively determine 
the intent of surgery for the study cohort. The study period ended one year prior to the release of REGATTA trial results [47]. This trial failed to show a survival advantage and highlighted the negative impact of gastrectomy in the setting of non-curable gastric cancer. Thus, it is possible that some gastrectomies performed during the study period were non-curative, in which case function would be prioritized over oncologic targets including margin status and extent of lymphadenectomy. We attempted to mitigate this risk in several ways; we excluded all patients with emergency admissions, previous gastrectomies, gastrectomies occurring more than 6 months following diagnosis and those identified with metastatic disease in their surgical pathology reports or by validated metastatic algorithm.

Higher case volume can impact certain measures of quality of care but may not address all care structures necessary for ideal textbook recovery. Future quality improvement strategies should consider using case-mix adjusted Textbook Outcome rates as a surgical quality metric.

Acknowledgements This study is financially supported by the Sherif and Mary Lou Hanna Chair in Surgical Oncology at Sunnybrook Health Sciences Centre and would not have been possible without the support of the PRESTO Group and its collaborators. PRESTO Group: Jordan Levy, MD. Division of General Surgery, Department of Surgery and Institute of Health Policy, Management, and Evaluation, University of Toronto, Canada. Vaibhav Gupta, MD. Division of General Surgery, Department of Surgery and Institute of Health Policy, Management, and Evaluation, University of Toronto, Canada. Elmira Amirazodi, MD, MSc. Evaluative Clinical Sciences, Sunnybrook Research Institute, Toronto, Canada. Catherine Allen-Ayodabo, MD, MPH. Evaluative Clinical Sciences, Sunnybrook Research Institute, Toronto, Canada. Naheed Jivraj, MBBS, MSc. Department of Anesthesia and Institute of Health Policy, Management, and Evaluation, University of Toronto, Toronto, Canada. Yunni Jeong, MD. Division of General Surgery, Department of Surgery and Institute of Health Policy, Management, and Evaluation, University of Toronto, Canada. Laura E. Davis, MSc. Evaluative Clinical Sciences, Sunnybrook Research Institute, Toronto, Canada. James R. Connor, MD, PhD. Department of Laboratory Medicine and Pathobiology, University of Toronto; Mount Sinai Hospital, Toronto, Canada. Eugene Hseih, MD. Department of Laboratory Medicine and Pathobiology, University of Toronto; Sunnybrook Health Sciences Centre, Toronto, Canada. Jolie Ringash, MD, MSc. Department of Radiation Oncology and Institute of Health Policy, Management, and Evaluation, University of Toronto, Canada. Rinku Sutradhar, PhD. Institute for Clinical Evaluative Sciences and Institute of Health Policy, Management, and Evaluation, University of Toronto, Canada. Biniam Kidane, MD, MSc. Section of Thoracic Surgery, Department of Surgery, University of Manitoba. Gail E. Darling, MD. Department of Surgery and Institute of Health Policy, Management, and Evaluation, University of Toronto; Toronto General Hospital/University Health Network, Toronto, Canada. Alyson L. Mahar, PhD. Manitoba Centre for Health Policy and Department of Community Health Sciences, University of Manitoba, Canada. Natalie G. Coburn, MD, MPH. Division of General Surgery, Department of Surgery and Institute of Health Policy, Management, and Evaluation, University of Toronto; Sunnybrook Health Sciences Centre, Toronto, Canada.

\section{References}

1. Rhome RM, et al. Predictors of positive margins after definitive resection for gastric adenocarcinoma and impact of adjuvant therapies. Int J Radiat Oncol Biol Phys. 2017;98(5):1106-15.

2. Ecker BL, et al. Minimally invasive gastrectomy for gastric adenocarcinoma in the United States: utilization and short-term oncologic outcomes. J Surg Oncol. 2015;112(6):616-21.

3. Mahar A, et al. Geographic variation in surgical practice patterns and outcomes for resected, non-metastatic gastric cancer across Ontario. Curr Oncol. 2018;25:e436.

4. Coburn NG, et al. Significant regional variation in adequacy of lymph node assessment and survival in gastric cancer. Cancer. 2006;107(9):2143-51.

5. Baxter NN, Tuttle TM. Inadequacy of lymph node staging in gastric cancer patients: a population-based study. Ann Surg Oncol. 2005;12(12):981.

6. Karna Sura HY, et al. How many lymph nodes are enough?defining the extent of lymph node dissection in stage I-III gastric cancer using the national cancer database. J Gastrointest Oncol. 2018;9(6):1168.

7. Bartlett EK, et al. Morbidity and mortality after total gastrectomy for gastric malignancy using the American college of surgeons national surgical quality improvement program database. Surgery. 2014;156(2):298-304.

8. Callahan MA, et al. Influence of surgical subspecialty training on in-hospital mortality for gastrectomy and colectomy patients. Ann Surg. 2003;238(4):629.

9. Hannan EL, et al. The influence of hospital and surgeon volume on in-hospital mortality for colectomy, gastrectomy, and lung lobectomy in patients with cancer. Surgery. 2002;131(1):6-15.

10. Smith DL, et al. Factors influencing the volume-outcome relationship in gastrectomies: a population-based study. Ann Surg Oncol. 2007;14(6):1846-52.

11. Birkmeyer JD, et al. Hospital volume and surgical mortality in the United States. N Engl J Med. 2002;346(15):1128-37.

12. de Gara CJ, Hanson J, Hamilton S. A population-based study of tumor-node relationship, resection margins, and surgeon volume on gastric cancer survival. Am J Surg. 2003;186(1):23-7.

13. Busweiler $\mathrm{L}$, et al. Textbook outcome as a composite measure in oesophagogastric cancer surgery. Br J Surg. 2017;104(6):742-50.

14. Benchimol EI, et al. The reporting of studies conducted using observational routinely-collected health data (RECORD) statement. PLoS Med. 2015;12(10):e1001885.

15. Robles SC, et al. An application of capture-recapture methods to the estimation of completeness of cancer registration. J Clin Epidemiol. 1988;41(5):495-501.

16. Clarke EA, Marrett LD, Kreiger N. Cancer registration in ontario: a computer approach. In: Jensen OM, editor. Cancer registration principles and methods. 95th ed. Lyon: IARC; 1991. p. 246-57.

17. Mahar AL, et al. Validating an algorithm to identify metastatic gastric cancer in the absence of routinely collected TNM staging data. BMC Health Serv Res. 2018;18(1):309.

18. Eskander A, et al. Volume-outcome relationships for head and neck cancer surgery in a universal health care system. Laryngoscope. 2014;124(9):2081-8.

19. Kulkarni GS, et al. Varied definitions of hospital volume did not alter the conclusions of volume-outcome analyses. J Clin Epidemiol. 2009;62(4):400-7.

20. Finlayson EV, Goodney PP, Birkmeyer JD. Hospital volume and operative mortality in cancer surgery: a national study. Arch Surg. 2003;138(7):721-5 (Discussion 726).

21. Austin PC, et al. Using the Johns Hopkins aggregated diagnosis groups (ADGs) to predict mortality in a general adult population cohort in Ontario, Canada. Med Care. 2011;49(10):932-9. 
22. Matheson FI, et al. Development of the Canadian Marginalization Index: a new tool for the study of inequality. Can J Public Health. 2012;103:S12-6.

23. Hosmer DW Jr, Lemeshow S, Sturdivant RX. Applied logistic regression, vol. 398. Hoboken: Wiley; 2013.

24. Juurlink D, et al. Canadian institute for health information discharge abstract database: a validation study. Toronto: Institute for Clinical Evaluative Sciences; 2006.

25. Canadian Institute for Health Information. CIHI data quality study of the 2009-2010 discharge abstract database. 2012. p. 1-139.

26. ICES. ICES Data Dictionary. Available from: https://www.cance rcareontario.ca/en/data-book-reporting-standards. Accessed 29 April 2019

27. Ghaferi AA, Birkmeyer JD, Dimick JB. Hospital volume and failure to rescue with high-risk surgery. Med care. 2011;49:1076-81.

28. Scott K, Grace R. Detection of lymph node metastases in colorectal carcinoma before and after fat clearance. Br J Surg. 1989;76(11):1165-7.

29. Smith AJ, et al. Multimodal CME for surgeons and pathologists improves colon cancer staging. J Cancer Educ. 2003;18(2):81-5.

30. Birkmeyer JD, et al. Hospital volume and late survival after cancer surgery. Ann Surg. 2007;245(5):777.

31. Finlayson EV, Goodney PP, Birkmeyer JD. Hospital volume and operative mortality in cancer surgery: a national study. Arch Surg. 2003;138(7):721-5.

32. Gordon TA, et al. Complex gastrointestinal surgery: impact of provider experience on clinical and economic outcomes1. J Am Coll Surg. 1999;189(1):46-56.

33. Bilimoria KY, et al. Effect of hospital type and volume on lymph node evaluation for gastric and pancreatic cancer. Arch Surg. 2008;143(7):671-8.

34. The Expert Panel on HPB Surgical Oncology. Hepatic, pancreatic, and biliary tract (HPB) surgical oncology standards. Program in Evidence-based Care Practice Guideline Report No.: 17-2 Version 2. Toronto: Cancer Care Ontario; 2006.

35. The Expert Panel on Thoracic Surgical Oncology. Thoracic surgical oncology standards. Program in Evidence-Based Care Practice Guideline Report No.: 17-1 Version 2. Toronto: Cancer Care Ontario; 2005.

36. Urbach DR, Baxter NN. Does it matter what a hospital is "high volume" for? Specificity of hospital volume-outcome associations for surgical procedures: analysis of administrative data. BMJ. 2004;328(7442):737-40.

37. Ghaferi AA, Birkmeyer JD, Dimick JB. Complications, failure to rescue, and mortality with major inpatient surgery in Medicare patients. Ann Surg. 2009;250(6):1029-34.

38. Brar SS, et al. Processes of care in the multidisciplinary treatment of gastric cancer: results of a RAND/UCLA expert panel. JAMA Surg. 2014;149(1):18-25.

39. Parikh D, et al. D2 gastrectomy: lessons from a prospective audit of the learning curve. Br J Surg. 1996;83(11):1595-9.

40. Kim CY, et al. Learning curve for gastric cancer surgery based on actual survival. Gastric Cancer. 2016;19(2):631-8.

41. Langer B. Role of volume outcome data in assuring quality in HPB surgery. HPB. 2007;9(5):330-4.

42. Shen JG, et al. Intraoperative frozen section margin evaluation in gastric cancer of the cardia surgery. Hepatogastroenterol. 2006;53(72):976-8.

43. Smyth EC, et al. Gastric cancer: eSMO clinical practice guidelines for diagnosis, treatment and follow-up. Ann Oncol. 2016;27(suppl 5):v38-49.

44. Ajani JA, et al. Gastric cancer, Version 3.2016, NCCN clinical practice guidelines in oncology. J Natl Compr Cancer Netw. 2016;14(10):1286-312.

45. Hardwick RH. D2 gastrectomy course, The royal college of surgeons of england, February 13-15, 2002. Gastric Cancer. 2002;5(4):0244-5.

46. Coburn N, et al. Staging and surgical approaches in gastric cancer: a clinical practice guideline. Curr Oncol. 2017;24(5):324.

47. Fujitani K, et al. Gastrectomy plus chemotherapy versus chemotherapy alone for advanced gastric cancer with a single non-curable factor (REGATTA): a phase 3, randomised controlled trial. Lancet Oncol. 2016;17(3):309-18.

Publisher's Note Springer Nature remains neutral with regard to jurisdictional claims in published maps and institutional affiliations.

\section{Affiliations}

\section{Jordan Levy ${ }^{1}$ (D) Vaibhav Gupta ${ }^{1}$. Elmira Amirazodi ${ }^{2}$. Catherine Allen-Ayodabo ${ }^{2}$. Naheed Jivraj ${ }^{3}$ - Yunni Jeong ${ }^{1}$. Laura E. Davis ${ }^{2}$. Alyson L. Mahar ${ }^{4}$. Charles De Mestral ${ }^{5,6}$. Olli Saarela ${ }^{7} \cdot$ Natalie Coburn $^{1,8}$. on behalf of the PRESTO Group}

1 Division of General Surgery, Department of Surgery and Institute of Health Policy, Management, and Evaluation, University of Toronto, Toronto, Canada

2 Evaluative Clinical Sciences, Sunnybrook Research Institute, Toronto, Canada

3 Department of Anesthesia and Institute of Health Policy, Management, and Evaluation, University of Toronto, Toronto, Canada

4 Manitoba Centre for Health Policy and Department of Community Health Sciences, University of Manitoba, Toronto, Canada
5 Division of Vascular Surgery, Department of Surgery and Institute of Health Policy, Management, and Evaluation, University of Toronto, Toronto, Canada

6 Li Ka Shing Knowledge Institute and St. Michael's Hospital, Toronto, Canada

7 Dalla Lana School of Public Health, University of Toronto, Toronto, Canada

8 Sunnybrook Health Sciences Centre, T2-11, 2075 Bayview Avenue, Toronto, ON M4N 3M5, Canada 